\title{
Effects of CDC42 on the proliferation and invasion of gastric cancer cells
}

\author{
DONG-SHU DU ${ }^{1 *}$, XIAO-ZHONG YANG ${ }^{2 *}$, QIONG WANG $^{2}$, WEI-JIE DAI ${ }^{2}$, \\ WEN-XIA KUAI ${ }^{2}$, YE-LIU LIU ${ }^{3}$, DECHANG CHU ${ }^{4}$ and XIAO-JUN TANG ${ }^{3}$ \\ ${ }^{1}$ Laboratory of Neuropharmacology and Neurotoxicology, Shanghai Key Laboratory of Bio-Energy Crops, \\ College of Life Science, Shanghai University, Shanghai 200444; Departments of ${ }^{2}$ Digestion and ${ }^{3}$ Gastrointestinal Surgery, \\ Huai'an First People's Hospital, Nanjing Medical University, Huai'an, Jiangsu 223300; \\ ${ }^{4}$ Department of Life Science, Heze University, Heze, Shandong 274015, P.R. China
}

Received December 23, 2014; Accepted September 22, 2015

DOI: $10.3892 / \mathrm{mmr} .2015 .4523$

\begin{abstract}
Cell division cycle 42 (CDC42), which is a member of the Rho GTPase family, has been reported to regulate the metastasis of various human cancer cells; however, the role of CDC42 in gastric cancer (GC) remains unclear. The present study aimed to investigate the effects of CDC42 on the proliferation, migration and invasion of GC. Furthermore, the molecular mechanisms underlying the effects of $\mathrm{CDC} 42$ on GC were explored. The expression levels of CDC42 in the AGS and SGC7901 human GC cell lines were reduced by RNA interference. Knockdown of CDC42 significantly inhibited the proliferation of AGS and SGC7901 cells, and it was suggested that this inhibitory process may be due to cell cycle arrest at G1/S phase and downregulation of cyclin A, cyclin D1, cyclin $\mathrm{E}$ and proliferating cell nuclear antigen. Furthermore, knockdown of CDC42 markedly inhibited the migration and invasion of GC cells, and suppressed the expression of matrix metalloproteinase 9. These results indicated that CDC42 is a
\end{abstract}

Correspondence to: Professor Xiao-Jun Tang, Department of Gastrointestinal Surgery, Huai'an First People's Hospital, Nanjing Medical University, 6 Beijing Road West, Huai'an, Jiangsu 223300 , P.R. China

E-mail:sdhzdds@126.com

Professor Dechang Chu, Department of Life Science, Heze University, 2269 Daxue Road, Heze, Shandong 274015, P.R. China E-mail: 357497709@qq.com

${ }^{*}$ Contributed equally

Abbreviations: CDC42, cell division cycle 42; GC, gastric cancer; PCNA, proliferating cell nuclear antigen, siRNA, small interfering RNA; FBS, fetal bovine serum; RT-qPCR, reverse transcription-quantitative PCR; GAPDH, glyceraldehyde-3-phosphate dehydrogenase; ECL, enhanced chemiluminescence; MMP9, matrix metalloproteinase 9

Key words: CDC42, gastric cancer, proliferation, migration, cell cycle, invasion key regulator involved in regulating the proliferation, migration and invasion of GC, and it may be considered a potential therapeutic target in GC.

\section{Introduction}

Gastric cancer (GC) is one of the most common causes of cancer-associated mortality worldwide, and often progresses to peritoneal metastasis (1). Numerous genetic and epigenetic alterations occur in the process of carcinogenesis and the progression of GC (2). The severity of GC may be associated with activation of oncogenes, inactivation of tumor suppressor genes, and deregulation of growth factors or their receptors. CDC42 is a small guanosine triphosphatase (GTPase) of the Rho family, which regulates the cell migration of various types of human cancer. At present, the role of cell division cycle 42 (CDC42) in the development of GC remains unclear.

Previous studies have reported the incidence of dysregulated expression of Rho GTPase family members and their effector proteins in various types of cancer, thus suggesting that these proteins may be involved in cancer initiation, progression and metastasis $(3,4)$. CDC42 is a member of the Rho family of small GTPases, and is considered a molecular switch of cell differentiation. CDC42 converts from its inactive GDP-bound form to the active GTP-bound form in response to diverse stimuli (5). The exact role of CDC42 in GC has yet to be elucidated; however, it is considered to positively regulate cancer cell growth, migration and invasion (6). A previous study demonstrated that a marked decrease in the levels of active CDC42 was correlated with the highly invasive potential of cell lines established from metastatic sites of colorectal adenocarcinoma (7). Furthermore, CDC42 has been shown to exert negative regulatory effects on intrinsic migration/invasion, and induce potentially relevant changes in the phosphorylation of protein kinase $\mathrm{C} \delta$, extracellular signal-regulated kinases $1 / 2$ and protein kinase A in aggressive breast cancer cells (8). CDC42 has been implicated to have an important role in colon and breast cancer; however, the regulatory effects and the underlying mechanisms of CDC42 in GC remain unknown. Based on previous studies $(9,10)$, the authors of the present study hypothesize that CDC42 may 
have an important role in GC metastasis and participate in regulating the migration and invasion of $\mathrm{GC}$ cells. In addition, CDC42 expression may be correlated with GC metastasis. The present study aimed to examine the association between CDC42 and the biological behavior of GC. These results may improve understanding regarding the mechanism underlying the invasion and metastasis of GC.

\section{Materials and methods}

Cell culture and reagents. The AGS and SGC7901 human GC cell lines were purchased from the Cell Bank of the Chinese Academy of Sciences (Shanghai, China). The cells were maintained in RPMI-1640 medium (Invitrogen; Thermo Fisher Scientific, Inc., Waltham, MA, USA) supplemented with $10 \%$ fetal bovine serum (FBS; Invitrogen), $100 \mathrm{IU} / \mathrm{ml}$ penicillin and $100 \mu \mathrm{g} / \mathrm{ml}$ streptomycin (Invitrogen) at $37^{\circ} \mathrm{C}$ in an atmosphere containing $5 \% \mathrm{CO}_{2}$.

Small interfering RNA (siRNA) synthesis and transient transfection. siRNA duplex oligonucleotides specifically targeting the human CDC42 cDNA sequence were synthesized, as follows: Forward 5'-GAAACUUGCCAAGAACAAAUU-3' and reverse 5'-UUUGUUCUUGGCAAGUUUCUU-3' (siCDC42). As a control, the following random siRNA sequences were used (Santa Cruz Biotechnology, Inc., Dallas, TX, USA): Forward 5'-UUCUCCGAACGUGUCACGU-3' and reverse 5'-ACGUGA CACGUUCGGAGAA-3' (siCon). Transient transfection of siCDC42 or siCon was performed using Lipofectamine ${ }^{\circledR} 2000$ (Invitrogen), according to the manufacturer's instructions. The cells were seeded at $1 \times 10^{5} /$ dish and cultured for $48 \mathrm{~h}$ prior to transfection with $100 \mathrm{nM}$ siRNA for $48 \mathrm{~h}$.

3-(4,5-dimethylthiazol-2-yl)-2,5-diphenyltetrazolium bromide (MTT) assay. Prior to transfection (1 day), $1 \times 10^{5}$ cells in $100 \mu \mathrm{l}$ growth medium were seeded in 96-well culture plates. The cells were transfected with siCDC42 or siCon at a final concentration of $100 \mathrm{nM}$. At 24, 48 or $72 \mathrm{~h}$ post-transfection, $100 \mu \mathrm{l}$ sterile MTT stock solution (1 mg/ml; Sigma-Aldrich, St. Louis, MO, USA) was added to each well. Following a $4 \mathrm{~h}$ incubation at $37^{\circ} \mathrm{C}$, the MTT solution was replaced with $200 \mu$ l dimethyl sulfoxide, followed by incubation for $8 \mathrm{~h}$. The absorbance was measured at a wavelength of $570 \mathrm{~nm}$ using a micro-enzyme-linked immunosorbent assay reader (Multiskan MK3; Thermo Labsystems, Franklin, MA, USA). Each sample was evaluated in triplicate.

Cell cycle analysis. The cells $\left(1 \times 10^{5}\right.$ cells/dish) were plated in 96-well plates and cultured for $24 \mathrm{~h}$, before being transfected with $100 \mathrm{nM}$ siCDC42 or siCon for $48 \mathrm{~h}$. For flow cytometry, the cells were trypsinized (Amresco, Solon, OH, USA), pelleted by centrifugation $(100 \mathrm{x} \mathrm{g}$ for $5 \mathrm{~min}$ ) and resuspended in $0.3 \mathrm{ml}$ of $0.1 \%$ Triton X-100 (Sigma-Aldrich)/phosphate-buffered saline. Subsequently, the cells were treated with RNase Type I-A (Sigma-Aldrich) at $37^{\circ} \mathrm{C}$ for $15 \mathrm{~min}$ and stained with propidium iodide (Invitrogen) for $10 \mathrm{~min}$. Cellular DNA content was determined using a FACSCalibur flow cytometer (BD Biosciences, San Jose, CA, USA). Cell cycle distribution was analyzed using ModFit LT (version 3.0; BD Biosciences) cell cycle analysis software. The experiment was performed three times, with each sample evaluated in triplicate.
Wound healing assay. The cells $\left(5 \times 10^{5}\right.$ cells/well) were transfected with $100 \mathrm{nM}$ siCDC42 or siCon for $24 \mathrm{~h}$, and were plated onto 6-well plates. Once the cells had reached $90 \%$ confluence, a single wound was created by gently scratching the attached cells with a sterile plastic pipette tip. The cells were then washed with serum-free medium, and wounded cell monolayers were allowed to heal for $24 \mathrm{~h}$. Images were captured under an optical microsope (Nikon, Tokyo, Japan) The experiment was performed three times, with each sample evaluated in triplicate.

In vitro invasion assay. The cells $\left(1 \times 10^{5}\right)$ were transfected with $100 \mathrm{nM}$ siCDC42 or siCon for $24 \mathrm{~h}$, and were then plated onto the upper side of BioCoat Matrigel Invasion Chambers (BD Biosciences). RPMI-1640 medium supplemented with $10 \%$ FBS was added to the lower chamber as a chemoattractant. Following a $24 \mathrm{~h}$ incubation, the cells on the upper surface of the Matrigel membrane were removed, and the invasive cells on the lower surface of the membrane were stained with $0.2 \%$ crystal violet (Invitrogen) in $10 \%$ ethanol. Five independent fields of invasive cells per well were observed under a phase contrast microscope (TS100; Nikon), and images were captured. The number of cells per field was counted and averaged. The experiment was performed three times, with each sample evaluated in triplicate.

RNA isolation and reverse transcription-quantitative polymerase chain reaction $(R T-q P C R)$. Total RNA was extracted from the cells using TRIzol ${ }^{\circledR}$ reagent (Invitrogen). First-strand cDNA was synthesized using $2.5 \mu \mathrm{g}$ RNA and AMV retroviridase (Promega Corporation, Madison, WI, USA). RT-qPCR was performed using the Bio-Rad iCycler $\mathrm{iQ}^{\mathrm{TM}}$ Real-Time PCR Detection system (Bio-Rad Laboratories, Inc., Hercules, CA, USA) and the following primers: CDC42, forward 5'-ACA TCTGTTTGTGGATAACTCA-3', reverse 5'-GGGAGCCAT ATACTCTTGGA-3'; and glyceraldehyde 3-phosphate dehydrogenase (GAPDH), forward 5'-CCACCCATGGCAAAT TCCATGGCA-3', and reverse 5'-TCTAGACGGCAGGTC AGGTCCACC-3'. The PCR mixture was prepared using SYBR Master mix (Takara Biotechnology Co., Ltd., Dalian, China), according to the manufacturer's protocol. To ensure that only the specific gene was amplified, a melting curve analysis was conducted at the end of each PCR experiment. Expression levels of each mRNA were determined using the ${ }^{\Delta \Delta} \mathrm{C}_{\mathrm{q}}$ method, with GAPDH used as an endogenous control.

Western blot analysis. For western blotting, the cells were lysed with Tris- $\mathrm{HCl}$ for $30 \mathrm{~min}$ on ice. The protein concentrations were determined using a bicinchoninic acid protein assay (Pierce Biotechnology, Inc., Rockford, IL, USA). The cell lysates (50 $\mu \mathrm{g}$ protein/lane) were separated by $10 \%$ sodium dodecyl sulfate-polyacrylamide gel electrophoresis and were transferred to nitrocellulose membranes (Hyclone; GE Healthcare Life Sciences, Logan, UT, USA). The membranes were then blocked with $5 \%(\mathrm{v} / \mathrm{v})$ skimmed milk and probed with primary antibodies at $4^{\circ} \mathrm{C}$ overnight. Following washing with phosphate-buffered saline, the membranes were incubated with polyclonal rabbit anti-mouse horseradish peroxidase-conjugated secondary antibodies (1:1,000 dilution; sc-358943; Santa Cruz Biotechnology, Inc.) at room temperature for $1 \mathrm{~h}$. The following primary 
A

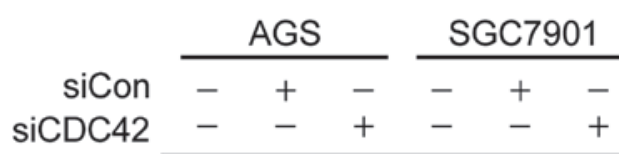

CDC42

$\beta$-actin
B

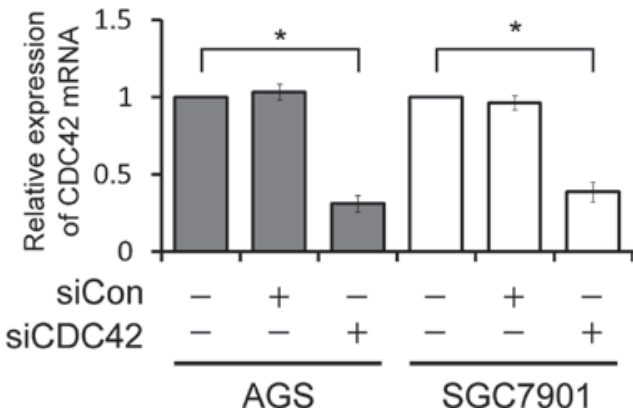

Figure 1. Efficiency of siCDC42 in AGS and SGC7901 human gastric cancer cell lines. The (A) protein and (B) mRNA expression levels of CDC42 were decreased in the AGS and SGC7901 cells following transfection with siCDC42. Data are presented as the mean \pm standard deviation. ${ }^{*}<0.05$ vs. the siCDC42-positive group. si, small interfering RNA; CDC42, cell division cycle 42; Con, control.
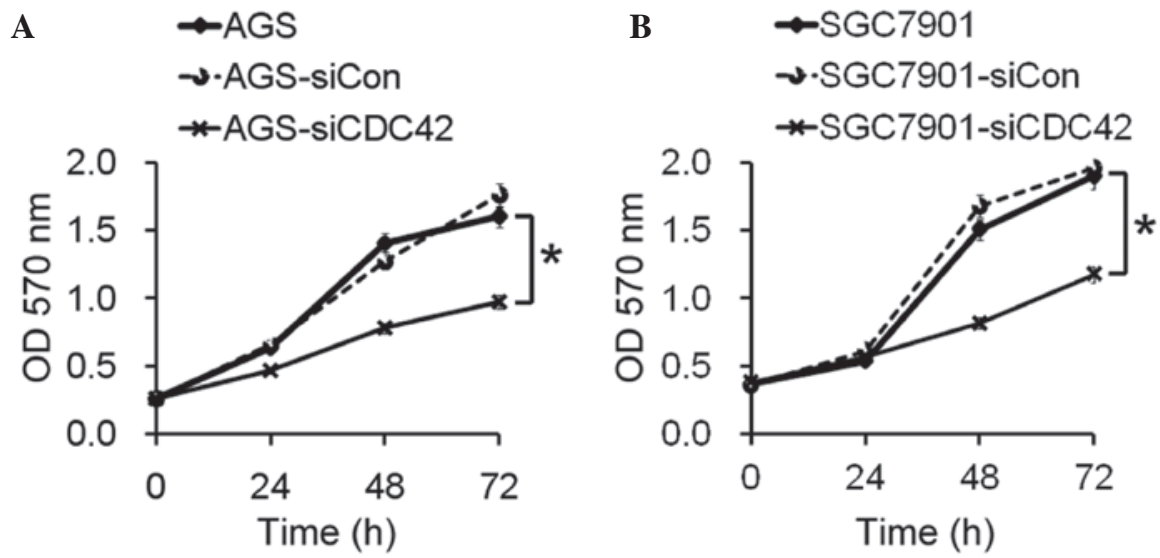

Figure 2. Inhibition of cell proliferation in AGS and SGC7901 human gastric cancer cells following CDC42 knockdown. CDC42 knockdown resulted in a significant inhibition of growth in the (A) AGS and (B) SGC7901 cells, as determined by an MTT assay. Data are presented as the mean \pm standard deviation. ${ }^{*} \mathrm{P}<0.05$ vs. the AGS-siCDC42 group. OD, optical density; si, small interfering RNA; CDC42, cell division cycle 42; Con, control.
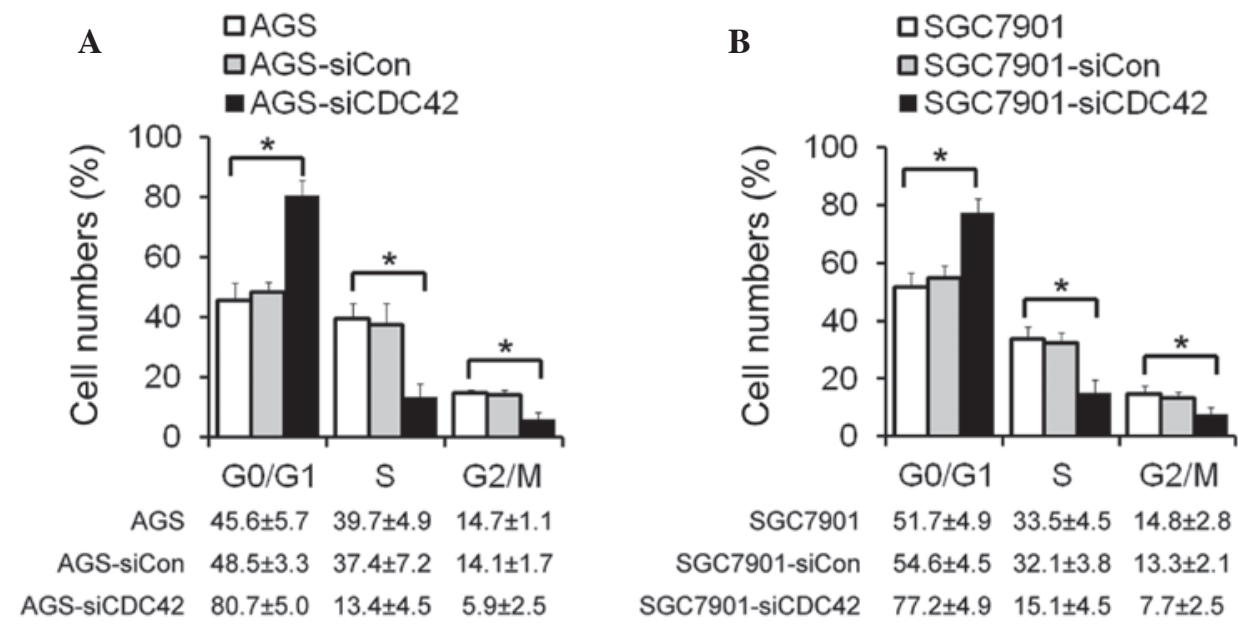

Figure 3. Cell cycle arrest of (A) AGS and (B) SGC7901 human gastric cancer cells following transfection with siCDC42, as compared with the control groups. Data are presented as the mean \pm standard deviation. " $\mathrm{P}<0.05$ vs. the siCDC42 positive group. si, small interfering RNA; CDC42, cell division cycle 42 ; Con, control.

antibodies, all polyclonal mouse anti-human purchased from Santa Cruz Biotechnology, Inc., were used in the present study: Anti-cyclin A (1:200 dilution; sc-271682), anti-cyclin B1 (1:300 dilution; sc-245), anti-cyclin D1 (1:300 dilution; sc-70899), anti-cyclin E (1:300 dilution; sc-247), anti-proliferating cell nuclear antigen (PCNA; 1:300 dilution; sc-71858), anti-matrix metalloproteinase 9 (MMP9; 1:300 dilution; sc-21733), anti- $\alpha$-tubulin (1:300 dilution; sc-23950), anti-CDC42 (1:250 dilution; sc-390210) and anti- $\beta$-actin (1:1,000 dilution; sc-8432). The bound antibodies were visualized using an enhanced chemiluminescence system (GE Healthcare Life Sciences, Chalfont, UK). 
A

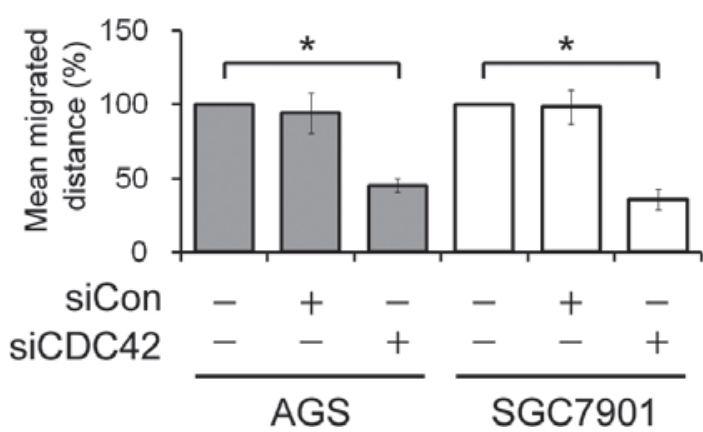

B

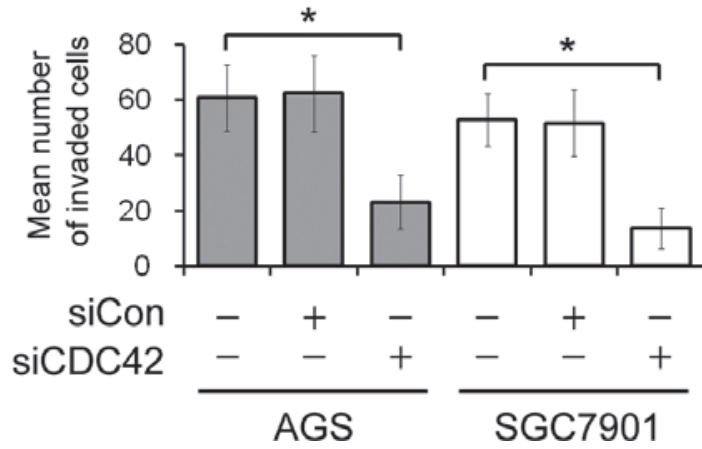

Figure 4. Effects of CDC42 knockdown on the migration and invasion of AGS and SGC7901 human gastric cancer cells. (A) Inhibitory effects of siCDC42 on the migration of AGS and SGC7901 cells, as determined by a wound healing assay. The data indicate the mean migrated distance $24 \mathrm{~h}$ after wound formation, and are presented as a percentage of the untransfected control cells. (B) Inhibitory effects of siCDC42 on the invasion of AGS and SGC7901 cells, as determined by an in vitro invasive assay. The data indicate the mean number of invaded cells $24 \mathrm{~h}$ after cell seeding. Data are presented as the mean \pm standard deviation. ${ }^{*} \mathrm{P}<0.05$ vs. the siCDC42-positive group. si, small interfering RNA, CDC42, cell division cycle 42; Con, control.

Statistical analysis. All experiments were performed three times, with each sample evaluated in triplicate. SPSS 18.0 software (SPSS Inc., Chicago, IL, USA) was used for all statistical analyses. The data were analyzed by Student's t-test, and are expressed as the mean \pm standard deviation. $\mathrm{P}<0.05$ was considered to indicate a statistically significant difference.

\section{Results}

Effect of siCDC42. In order to elucidate the functional role of CDC42 in GC, the present study examined the effects of CDC42 expression knockdown on GC cell growth in vitro. AGS and SGC7901 cells were transfected with siCDC42 or siCon. Based on their high expression of $\mathrm{CDC} 42$, the human AGS and SGC7901 cell lines were selected for use as cell models in the present study. siCDC42 effectively suppressed the protein and mRNA expression levels of CDC42 in the GC cells, as determined by western blotting and RT-qPCR (Fig. 1).

Role of CDC42 in growth of GC cells. The present study aimed to determine the biological role of $\mathrm{CDC} 42$ in the growth of GC cells. The proliferative ability of the AGS and SGC7901 cells transfected with siCDC42 was significantly decreased, as compared with the siCon-transfected cells (Fig. 2).

Cell cycle distribution following siCDC42 transfection. The mechanisms underlying the effects of CDC42 on cell growth inhibition were also investigated. Cell cycle distribution of the AGS and SGC7901 cells was determined by flow cytometry. The number of cells in G0/G1 phase was increased, and the proportion of cells in S phase and G2/M phase was decreased in the siCDC42-transfected AGS and SGC7901 cells, as compared with the control cells (Fig. 3). These results suggest that the inhibitory effects of CDC42 knockdown on GC cell growth may be mediated by cell cycle arrest at G1/S phase.

Migration and invasion of GC cells following siCDC42 transfection. In vitro wound healing and invasive assays were performed to determine the effects of CDC42 knockdown on $\mathrm{GC}$ cell migration and invasion. The rate of wound closure in the siCDC42-transfected AGS and SGC7901 cells was delayed, as compared with the control cells (Fig. 4A). In addition, the

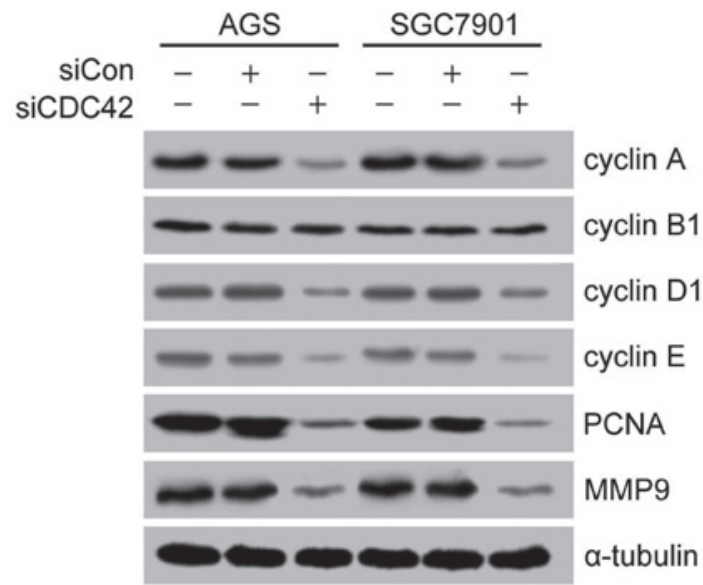

Figure 5. Effects of CDC42 knockdown on the expression levels of cell cycle related-proteins and MMP9 in AGS and SGC7901 human gastric cancer cells. The protein expression levels of cyclin A, cyclin B1, cyclin D1, cyclin E, PCNA, MMP9 and $\alpha$-tubulin were detected by western blotting. $\alpha$-tubulin was used as the loading control. si, small interfering RNA; CDC42, cell division cycle 42; Con, control; PCNA, proliferating cell nuclear antigen; MMP9, matrix metalloproteinase 9 .

number of invasive siCDC42-transfected AGS and SGC7901 cells was lower, as compared with the siCon-transfected AGS and SGC7901 cells (Fig. 4B).

Effect of siCDC42 on cell cycle protein levels. In order to investigate which signaling pathway mediated the suppressive effects of CDC42 on cell cycle arrest, cell cycle-related proteins, including cyclin A, cyclin D1, cyclin E, PCNA and MMP9 were detected by western blotting. As shown in Fig. 5, the protein expression levels of cyclin A, cyclin D1, cyclin E, PCNA and MMP9 were significantly reduced in the siCDC42-transfected cells, as compared with the siCon-transfected cells. However, the expression levels of cyclin B1 were not changed between the groups.

\section{Discussion}

Previous studies have demonstrated that CDC42 is overexpressed in lung (11-13), breast (14), testicular (15), colorectal (16) and esophageal (17) cancer. However, the 
effects and underlying mechanisms of CDC42 on GC remain unclear.

The present study investigated the effects of CDC42 on the proliferation, migration and invasion of GC cells. The results demonstrated that inhibition of CDC42 expression, via siCDC42 transfection, inhibited the proliferation, migration and invasion of AGS and SGC7901 GC cells. These findings suggested that CDC42 may be a potential target for GC treatment.

The present study aimed to investigate the molecular mechanisms underlying the effects of CDC42 knockdown on the inhibition of GC cell proliferation. The results of the western blot and MTT assays revealed that CDC42 knockdown inhibited the cell proliferation. In addition, in order to confirm whether cell cycle-associated proteins regulate the effects of CDC42 on GS-cell proliferation, the expression levels of cell cycle-regulatory proteins, including cyclin A, cyclin D1 and cyclin E, were downregulated in the siCDC42-transfected cells.

PCNA is a homotrimeric protein, which is essential for cell cycle progression, replication and DNA repair (18). A previous study indicated that PCNA functions as a proliferation marker, since it is expressed in late G1 phase and early $\mathrm{S}$ phase of the cell cycle (18). In the present study, knockdown of CDC42 resulted in the downregulation of PCNA. Therefore, the inhibitory effects of CDC42 knockdown may be mediated by cell cycle arrest at G1/S phase.

Metastasis refers to the spread of cancer from the original tumor site to other parts of the body, and is a characteristic of malignant cancer (19). A previous study demonstrated that CDC42 is an important regulator of metastasis in human cancer (3). MMPs degrade components of the extracellular matrix, and are involved in the regulation of development, growth and spread of primary tumors (20). The expression levels of MMP9, an important member of the MMP family, are significantly higher in GC tissue $(63.0 \%)$, as compared with in normal tissue (6.7\%), and the expression of MMP9 is associated with tumor size, depth of invasion, lymph node metastasis, degree of histological differentiation and pathological stage (21). In the present study, the expression of MMP9 was attenuated following transfection with siCDC42. These results indicated that the inhibitory effects of CDC42 knockdown on GC cell migration and invasion may be mediated by MMP9.

In conclusion, the present study demonstrated that siCDC42-induced suppression of CDC42 inhibited GC cell proliferation by arresting cells at $\mathrm{G} 1 / \mathrm{S}$ phase of the cell cycle, and reducing the expression of cyclin A, cyclin D1, cyclin E and PCNA. In addition, siCDC42 was shown to inhibit the migration and invasion of GC cells via downregulation of MMP9. These data suggest that CDC42 may be considered a promising target for the effective treatment of GC.

\section{Acknowledgements}

The present study was supported by the Chinese National Natural Science Foundation(grant nos.31100838 and31571171), the Nanjing Medical University Science and Technology Development Program (grant no. 09NJMUZ30), the Shanghai Natural Science Foundation (grant no. 15ZR1414900) and the Young Teachers of Shanghai Universities Training Program.

\section{References}

1. Krejs GJ: Gastric cancer: Epidemiology and risk factors. Dig Dis 28: 600-603, 2010.

2. Yasui W, Sentani K, Sakamoto N, Anami K, Naito Y and Oue N: Molecular pathology of gastric cancer: Research and practice. Pathol Res Pract 207: 608-612, 2011.

3. Karlsson R, Pedersen ED, Wang Z and Brakebusch C: Rho GTPase function in tumorigenesis. Biochim Biophys Acta 1796: 91-98, 2009.

4. Van der Meel R, Symons MH, Kudernatsch R, Kok RJ, Schiffelers RM, Storm G, Gallagher WM and Byrne AT: The VEGF/Rho GTPase signalling pathway: A promising target for anti-angiogenic/anti-invasion therapy. Drug Discov Today 16: 219-228, 2011.

5. Sinha S and Yang W: Cellular signaling for activation of Rho GTPase Cdc42. Cell Signal 20: 1927-1934, 2008.

6. Stengel $\mathrm{K}$ and Zheng Y: Cdc42 in oncogenic transformation, invasion, and tumorigenesis. Cell Signal 23: 1415-1423, 2011.

7. de Toledo M, Anguille C, Roger L, Roux P and Gadea G: Cooperative anti-invasive effect of Cdc42/Racl activation and ROCK inhibition in SW620 colorectal cancer cells with elevated blebbing activity. PLoS One 7: e48344, 2012.

8. Zuo Y, Wu Y and Chakraborty C: Cdc42 negatively regulates intrinsic migration of highly aggressive breast cancer cells. J Cell Physiol 227: 1399-1407, 2012.

9. Lee YC, Cheng TH, Lee JS, Chen JH, Liao YC, Fong Y, Wu CH and Shih YW: Nobiletin, a citrus flavonoid, suppresses invasion and migration involving FAK/PI3K/Akt and small GTPase signals in human gastric adenocarcinoma AGS cells. Mol Cell Biochem 347: 103-115, 2011.

10. Chen Q, Chen X, Zhang M, Fan Q, Luo S and Cao X: miR-137 is frequently down-regulated in gastric cancer and is a negative regulator of Cdc42. Dig Dis Sci 56: 2009-2016, 2011.

11. Chen QY, Jiao DM, Yao QH, Yan J, Song J, Chen FY, Lu GH and Zhou JY: Expression analysis of Cdc42 in lung cancer and modulation of its expression by curcumin in lung cancer cell lines. Int J Oncol 40: 1561-1568, 2012.

12. Zhang JY, Zhang D and Wang EH: Overexpression of small GTPases directly correlates with expression of $\delta$-catenin and their coexpression predicts a poor clinical outcome in nonsmall cell lung cancer. Mol Carcinog 52: 338-347, 2013.

13. Liu Y, Xu H, Liu N, Wang L and Wang E: Correlation of expression of p120ctn, RhoA, and $\mathrm{Cdc} 42$ and their significance in non-small cell lung cancer. Zhongguo Fei Ai Za Zhi 8: 304-308, 2005 (In Chinese).

14. Jiang LC, Zhang Y and Qu XC: Effects of Cdc42 overexpression on the estrogen-enhanced multidrug resistance in breast cancer cells. Zhonghua Zhong Liu Za Zhi 33: 489-493, 2011 (In Chinese).

15. Kamai T, Yamanishi T, Shirataki H, Takagi K, Asami H, Ito Y and Yoshida K: Overexpression of RhoA, Rac1, and Cdc42 GTPases is associated with progression in testicular cancer. Clin Cancer Res 10: 4799-4805, 2004.

16. Gómez Del Pulgar T, Valdés-Mora F, Bandrés E, Pérez-Palacios R, Espina C, Cejas P, García-Cabezas MA, Nistal M, Casado E, González-Barón M, et al: Cdc42 is highly expressed in colorectal adenocarcinoma and downregulates ID4 through an epigenetic mechanism. Int J Oncol 33: 185-193, 2008.

17. Liu Z, Feng JG, Tuersun A, Liu T, Liu H, Liu Q, Zheng ST, Huang CG, Lv GD, Sheyhidin I and Lu XM: Proteomic identification of differentially-expressed proteins in esophageal cancer in three ethnic groups in Xinjiang. Mol Biol Rep 38: 3261-3269, 2011.

18. Strzalka W and Ziemienowicz A: Proliferating cell nuclear antigen (PCNA): A key factor in DNA replication and cell cycle regulation. Ann Bot 107: 1127-1140, 2011.

19. Hanahan D and Weinberg RA: Hallmarks of cancer: The next generation. Cell 144: 646-674, 2011.

20. Bauvois B: New facets of matrix metalloproteinases MMP-2 and MMP-9 as cell surface transducers: Outside-in signaling and relationship to tumor progression. Biochim Biophys Acta 1825: 29-36, 2012.

21. Yang S, Zhao Z, Wu R, Lu H, Zhang X, Huan C, Wang C, Wu X and Guan G: Expression and biological relationship of vascular endothelial growth factor-A and matrix metalloproteinase-9 in gastric carcinoma. J Int Med Res 39: 2076-2085, 2011. 